\title{
Admissão ao Internato Complementar em Portugal: Análise dos Resultados do Exame Nacional de Seriação entre 2006 e 2011
}

\author{
Admission to Residence Training in Portugal: Analysis of the \\ National Exam Results between 2006 and 2011
}

Isabel Pavão MARTINS 1

Acta Med Port 2013 Sep-Oct;26(5):569-577

RESUMO

Introdução: Em Portugal os médicos recém-licenciados realizam anualmente um exame nacional de seriação para se habilitarem às vagas do internato da especialidade.

Objectivos: Neste estudo analisaram-se os resultados dos exames nacionais de seriação efectuados nos últimos 6 anos, para identificar os factores responsáveis pela classificação.

Material e Métodos: Foram analisados os dados demográficos (nacionalidade, género) e académicos (escola médica de origem, média de curso, ano do exame) de 8956 candidatos ao exame nacional de seriação e estudada a sua relação com a classificação obtida no exame. Efectuou-se ainda uma análise de regressão linear múltipla para identificar os factores determinantes da classificação.

Resultados: Encontraram-se diferenças significativas na proporção de candidatos licenciados pelas diferentes escolas nacionais e pequenas discrepâncias, embora significativas, na sua classificação média. A percentagem de licenciados no estrangeiro atingiu os $15 \%$ nos últimos dois anos. A média de curso, nacionalidade, escola de origem, ano do exame e o género influenciavam o resultado do exame nacional de seriação e explicavam $39 \%$ da sua variância, particularmente a média de curso. A correlação entre essas duas classificações variava entre 0,679 e 0,586 nas Escolas Nacionais mas era fraca nos licenciados no estrangeiro. As Faculdades Nacionais apresentavam diferenças entre 0,2 e 8,5 pontos no exame nacional de seriação.

Conclusão: A formação pré graduada (média de curso e escola de Licenciatura) relaciona-se com o desempenho no exame nacional de seriação, sobretudo nos Licenciados em Portugal. Estes dados constituem uma oportunidade para comparar resultados a nível nacional e para reflectir sobre o impacto do futuro exame nacional de seriação.

Palavras-chave: Avaliação Educacional; Educação Médica Pós-Graduada; Escolas Médicas; Internato Médico; Modelos Educacionais.

\section{ABSTRACT}

Introduction: In Portugal, new medical graduates undertake every year the Exame Nacional de Seriação, to be ranked for the available postgraduate residency posts within the National Health Service. Exame Nacional de Seriação is a multiple choice questions test on internal medicine.

Objectives: This study analyses the Exame Nacional de Seriação results between 2006 and 2011, to identify the variables that predict its score.

Material and Methods: Academic (graduating University, final classification) and biographic data (gender, nationality) of 8956 candidates were analyzed and related to the Exame Nacional de Seriação' score. A linear regression analysis was performed to determine the predictors of that score.

Results: There were significant differences in the proportion of candidates coming from each National Medical School attending the Exame Nacional de Seriação, and minor but significant discrepancies in their classification. The percentage of international medical graduates increased to $15 \%$ in the last two years. Candidates' gender, nationality (Portuguese or other), year of exam, graduating medical school and final graduating classification had a significant relation with ENS score, explaining 39\% of Exame Nacional de Seriação' variance. Final graduating classification was the single most predictive variable, which correlation with Exame Nacional de Seriação' score varied between .679 and .586 across Portuguese Medical Schools, but was weak among candidates graduated abroad.

Conclusion: Pregraduate training (Final Graduating Classification and Medical School) is related to Exame Nacional de Seriação' score, particularly among national graduates. This data might be relevant to understand the impact of the changes on Exame Nacional de Seriação about to be implemented.

Keywords: Education, Medical, Graduate; Educational Measurement; Internship and Residency; Models, Educational; Schools, Medical.

\section{INTRODUÇÃO}

O acesso ao internato complementar representa um marco importante na vida dos médicos pois dá inicio à sua diferenciação profissional, determina o local e a equipa com quem vão trabalhar nos 5-6 anos seguintes e garanteIhes um emprego.

Nos países onde existe Serviço Nacional de Saúde, a formação médica pós graduada faz-se maioritária ou exclusivamente nas Instituições públicas, embora o sistema de acesso e de selecção dos candidatos varie consoante os países, as especialidades e até os Centros Hospitalares / Unidades de Saúde. Na maioria dos países Europeus e na América do Norte, os serviços de acolhimento têm um papel muito activo na selecção dos candidatos, quer através de avaliações curriculares e exames de pré-selecção, quer mediante entrevistas individuais.

Em Portugal, o Ministério da Saúde e a Ordem dos Médicos certificam os serviços responsáveis pela formação pós graduada e o Ministério da Saúde decreta anualmente

1. Laboratório de Estudos de Linguagem. Departamento de Neurociências. Faculdade de Medicina e Instituto de Medicina Molecular. Universidade de Lisboa. Lisboa. Portugal. Recebido: 27 de Fevereiro de 2013 - Aceite: 11 de Maio de 2013 | Copyright $\odot$ Ordem dos Médicos 2013 
o número e o local das vagas para cada especialidade. Todavia, desde os anos 70, a selecção e a distribuição dos candidatos são inteiramente determinadas por uma única prova de admissão, igual para todas as especialidades e serviços, designada por Exame Nacional de Seriação (ENS). Este exame, com uma duração de 150 minutos, consiste num teste escrito com 100 perguntas de escolha múltipla, apenas com uma alínea correcta, divididas equitativamente por cinco áreas da Medicina Interna e com uma única referência bibliográfica, de um livro de texto Norte-americano. ${ }^{1}$

A classificação obtida neste teste estabelece a ordem pela qual os candidatos escolhem as vagas disponíveis, recorrendo-se à média de curso em situações de empate. Ou seja, o candidato escolhe o serviço em função da sua nota mas o serviço responsável pela formação não escolhe nem se pronuncia sobre o candidato, o que dá particular relevância a este exame de acesso à especialidade.

O ENS foi instituído exactamente para ordenar a admissão dos médicos ao internato complementar através de um método equitativo. Porém, a selecção dos conteúdos para este teste foi feita de forma pouco criteriosa, fazendo prevalecer o número de capítulos e páginas sobre a pertinência dos temas. De facto, a matéria seleccionada induz o médico a memorizar uma extensa lista de dados e detalhes sobre doenças raras que não fazem parte da clínica de rotina e são irrelevantes para a sua actividade profissional mas, por outro lado, permite-lhe ignorar patologias tão frequentes e importantes como a diabetes, as doenças oncológicas mais comuns em Portugal, a depressão, o coma, as doenças infecciosas banais e as urgências cirúrgicas, para mencionar apenas alguns tópicos da medicina do adulto.

A falta de critério na selecção dos conteúdos traduz-se assim num enorme desperdício do potencial de aprendizagem. Sabe-se que a avaliação modela a preparação e a estratégia cognitiva dos alunos/candidatos ${ }^{2-4}$ e isso é particularmente importante num exame decisivo para as suas vidas. Embora o ENS pudesse estimular, a nível nacional, uma aprendizagem orientada para a prática clínica e uma revisão aprofundada de conhecimentos, raciocínios e atitudes após a formação pré graduada, o actual formato acaba por promover uma aprendizagem mais superficial e dirigida ao sucesso no exame e não à aquisição de conhecimentos em si.

Apesar das críticas repetidas e do carácter limitado dos conteúdos e da bibliografia, o exame manteve-se inalterado ao longo de mais de três décadas. Além disso, tanto quanto sabemos, nunca foi divulgada uma análise aprofundada dos resultados, pelo menos fora do Ministério da Saúde. Independentemente do seu objectivo, este exame tem aspectos muito particulares pois é o único que avalia os médicos a nível Nacional (o que o torna próximo de um Exame de Estado), é o primeiro exame a que os recém-licenciados se submetem fora das Faculdades e é tutelado pelas entidades de Saúde, com consequências sobre a formação pós graduada.

Encontrando-se neste momento em preparação e dis- cussão um novo exame de seriação, pareceu-nos que seria interessante e oportuno analisar os resultados dos últimos exames realizados e compreender os factores que determinam o seu resultado. Para além do seu interesse intrínseco, esta análise poderá ser comparada com os resultados do futuro exame de seriação.

\section{MATERIAL E MÉTODOS}

A análise foi efectuada numa base de dados, amavelmente cedida pela Administração Central do Sistema de Saúde (ACSS). Esta base incluía alguns dados biográficos (género, nacionalidade) e académicos dos candidatos (Faculdade de Licenciatura e média de curso), além dos resultados obtidos no ENS. Os dados correspondem aos dos exames realizados, na primeira fase, entre 2006 e 2011, cujos candidatos foram admitidos no internato complementar entre Janeiro de 2007 e Janeiro de 2012. A identidade dos médicos participantes foi apagada da base, embora a respectiva classificação individual seja pública e disponibilizada todos os anos através do site do Ministério da Saúde.

\section{Análise estatística}

A distribuição das notas de licenciatura e do exame de seriação foi estudada pelo teste de Kolmogorov-Smirnov. As comparações entre grupos foram feitas pelo test $t$ de Student ou por Análises de Variância (ANOVA) com análises pós hoc de Tukey HSD. A associação entre variáveis foi estudada pelo teste do $c^{2}$ (variáveis nominais) ou através de correlações de Pearson (variáves lineares). Para identificar os factores determinantes da classificação no ENS foi realizada uma análise de regressão linear múltipla, sendo a nota do exame a variável dependente e a nota de curso, nacionalidade (Portuguesa ou estrangeira), género e Faculdade de origem, as variáveis independentes. Esta análise permite determinar o efeito de cada uma das variáveis (através do coeficiente de regressão) no resultado do ENS, tendo em conta as interacções entre todas as variáveis testadas no modelo e determinar a percentagem da classificação no ENS determinada por essas variáveis. Foram realizados testes de diagnóstico de multi-colinearidade e de auto correlação dos erros (estatística de Durbin-Watson). O nível de significância foi estabelecido para um valor de $p<0,05$.

A análise foi efectuada num programa de estatística SPSS ${ }^{5}$ versão 21.0, após conversão de ficheiros de Excell.

\section{RESULTADOS}

\section{Distribuição dos candidatos por ano e por Faculdade de Licenciatura}

Entre 2006 e 2011 foram realizados, na primeira fase, 8956 exames nacionais de seriação. O menor número de candidatos verificou-se em 2007, seguido por um aumento anual dos candidatos até 2011 (Tabela 1). Em 2006, houve dois cursos consecutivos a realizar este exame, devido à redução do internato geral em seis meses, o que explica o elevado número de candidatos nesse ano.

A distribuição dos candidatos pelas Escolas Médicas 
Tabela 1 - Distribuição dos candidatos por ano e por Faculdade de Licenciatura

\begin{tabular}{|c|c|c|c|c|c|c|c|}
\hline \multicolumn{7}{|c|}{ Ano Candidatura } & \multirow[t]{2}{*}{ Total } \\
\hline & 2006 & 2007 & 2008 & 2009 & 2010 & 2011 & \\
\hline \multirow{2}{*}{ FMUL } & 366 & 249 & 258 & 227 & 328 & 357 & 1835 \\
\hline & $22,6 \%$ & $20,1 \%$ & $20,3 \%$ & $19,2 \%$ & $20,6 \%$ & $19,9 \%$ & $20,5 \%$ \\
\hline \multirow{2}{*}{ FCML } & 317 & 203 & 202 & 231 & 208 & 239 & 1400 \\
\hline & $19,6 \%$ & $16,4 \%$ & $15,9 \%$ & $16,0 \%$ & $13,1 \%$ & $13,3 \%$ & $15,6 \%$ \\
\hline \multirow{2}{*}{ FMUC } & 217 & 233 & 242 & 288 & 258 & 305 & 1597 \\
\hline & $16,7 \%$ & 18,8 & $19,1 \%$ & $19,9 \%$ & $16,2 \%$ & $17,0 \%$ & $17,8 \%$ \\
\hline \multirow{2}{*}{ FMUP } & 294 & 207 & 211 & 248 & 241 & 271 & 1472 \\
\hline & $18,1 \%$ & 16,7 & $16,6 \%$ & $17,2 \%$ & $15,2 \%$ & $15,1 \%$ & $16,4 \%$ \\
\hline \multirow{2}{*}{ ICBAS } & 202 & 117 & 156 & 136 & 173 & 188 & 972 \\
\hline & $12,5 \%$ & 9,4 & $12,3 \%$ & $9,4 \%$ & $10,9 \%$ & $10,5 \%$ & $10,9 \%$ \\
\hline \multirow{2}{*}{ UCBI } & 0 & 53 & 37 & 58 & 77 & 84 & 309 \\
\hline & $0,0 \%$ & $4,3 \%$ & $2,9 \%$ & $4,0 \%$ & $4,8 \%$ & $4,7 \%$ & $3,5 \%$ \\
\hline \multirow{2}{*}{ UM - ECS } & 0 & 48 & 37 & 55 & 60 & 64 & 264 \\
\hline & $0,0 \%$ & $3,9 \%$ & $2,9 \%$ & $3,8 \%$ & $3,8 \%$ & $3,6 \%$ & $2,9 \%$ \\
\hline \multirow{2}{*}{$\begin{array}{l}\text { Faculdades } \\
\text { Estrangeiras }\end{array}$} & 171 & 130 & 127 & 152 & 244 & 283 & 1107 \\
\hline & $10,5 \%$ & $10,5 \%$ & $10,0 \%$ & $10,5 \%$ & $15,4 \%$ & $15,8 \%$ & $12,4 \%$ \\
\hline \multirow[t]{2}{*}{ Total } & 1621 & 1240 & 1270 & 1445 & 1589 & 1791 & 8956 \\
\hline & $100 \%$ & $100 \%$ & $100 \%$ & $100 \%$ & $100 \%$ & $100 \%$ & $100 \%$ \\
\hline
\end{tabular}

Nacionais foi bastante heterogénea, embora estável ao longo dos seis anos (Tabela 1). Cerca de um quinto dos médicos tinham completado a Licenciatura/Mestrado Integrado na Faculdade de Medicina da Universidade de Lisboa (FMUL), seguindo-se por ordem decrescente as Faculdades de Medicina da Universidade de Coimbra (FMUC), Faculdade de Medicina da Universidade do Porto (FMUP), a Faculdade de Ciências Médicas de Lisboa (FCM) e o Instituto de Ciências Biomédicas Abel Salazar (ICBAS) da Universidade do Porto. Os médicos licenciados pela Faculdade de Ciências da Saúde da Universidade da Beira Interior (UBI) e pela Escola de Ciências da Saúde da Universidade do Minho (UM) só participaram neste exame a partir de 2007 e constituem 4 e 3\% dos candidatos.

Por outro lado, $12,4 \%$ dos candidatos tinham-se licenciado no estrangeiro (Licenciados Médicos Internacionais). Este grupo aumentou de forma significativa nos últimos dois anos, passando de 10 para $15 \%$ do total de candidatos e, em 2011, o seu número ultrapassou os candidatos de cinco das sete Faculdades Nacionais (Tabela 1).

\section{Análise das variáveis demográficas e académicas}

No total dos seis anos, verificou-se um predomínio significativo de candidatos Portugueses $(92,3 \%)$, do sexo feminino $(63,3 \%)$ e de Licenciados em Universidades Por- tuguesas (87,6 \%). A distribuição entre géneros sofreu variações anuais, embora sempre com predomínio feminino. Os médicos estrangeiros constituíam $8.47 \%(n=759)$ do total de candidatos, com uma percentagem que variou entre 6,2 e $9,2 \%$ ao longo dos 6 anos. Embora a maioria $(75,8 \%)$ dos estrangeiros se tivesse licenciado fora de Portugal, 24,2\% completaram a sua Licenciatura/Mestrado integrado em Universidades Portuguesas, constituindo uma percentagem variável dos candidatos das diversas escolas. Essa percentagem era mais alta na Universidade de Coimbra ( $4,1 \%$ dos candidatos da FMUC eram estrangeiros) e no ICBAS $(3,2 \%)$, e mais baixa $(0,3 \%)$ na UBI e nula na UM.

Os candidatos estrangeiros (considerando aqui quer os Licenciados no estrangeiro, quer em Portugal) eram originários de mais de 50 países. Os grupos de maior dimensão, no total dos 6 anos e por ordem decrescente, eram provenientes da Ucrânia ( $n=143 ; 18,84 \%)$, Espanha ( $n=$ $97 ; 12,8 \%)$, Brasil $(n=72)$, Rússia $(n=51)$, Guiné Bissau $(n=46)$, Cabo Verde $(n=38)$, Moldávia $(n=38)$, Angola ( $n=31)$, Roménia $(n=29)$, Itália $(n=25)$, Moçambique $(n=13)$, Venezuela $(n=13)$, Alemanha $(n=11)$ Colômbia $(n=10)$, tendo os restantes países menos de 10 candidatos. No total, os médicos da União Europeia representavam $18,4 \%(n=140)$ de todos os estrangeiros e os de países de 

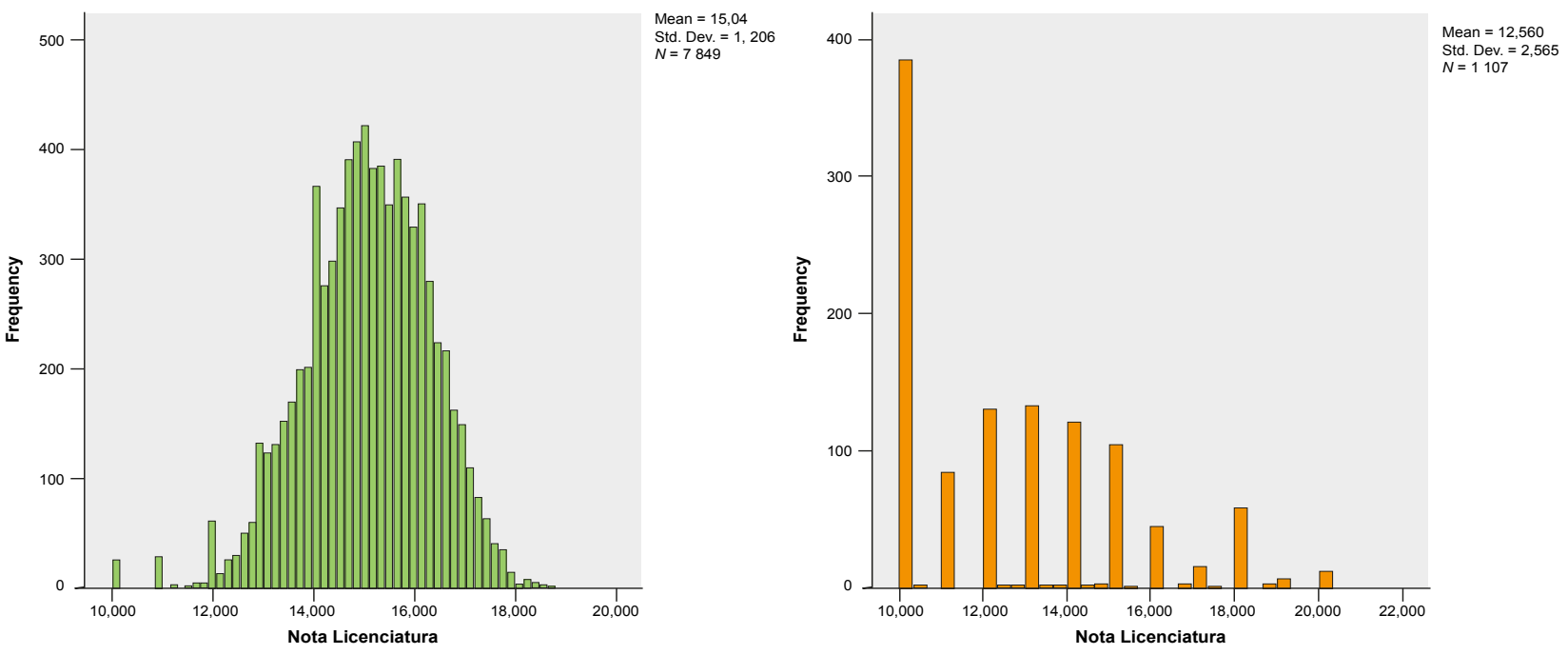

Figura 1 - Histograma da distribuição das médias de Licenciatura: a) dos candidatos Licenciados em Portugal e b) dos Licenciados Médicos Internacionais. A média de Licenciatura não seguiu uma distribuição normal (K-S Z = 8,52, $p<0,000)$.

Tabela 2 - Média de curso, classificação média no exame de seriação e correlação entre as duas notas, por Faculdade.

\begin{tabular}{|c|c|c|c|}
\hline Faculdade de Licenciatura/ Mestrado Integrado & $\begin{array}{l}\text { Média de curso } \\
\text { Média } \pm \text { (DP) } \\
2006-2011\end{array}$ & $\begin{array}{l}\text { Classificação } \\
\text { no Exame } \\
\text { Média } \pm \text { (DP) } \\
2006-2011\end{array}$ & $\begin{array}{l}\text { Correlação } \\
\text { Nota curso } \\
\text { Nota de exame } \\
\text { ( } r \text { de Pearson) }\end{array}$ \\
\hline $\begin{array}{l}\text { Universidade de Lisboa } \\
(n=1835)\end{array}$ & $\begin{array}{l}15,70 \\
(1,03)\end{array}$ & $\begin{array}{r}69,41 \\
(14,36)\end{array}$ & $0,648^{* *}$ \\
\hline $\begin{array}{l}\text { Universidade Nova de Lisboa (Faculdade de Ciências Médicas) } \\
(n=1400)\end{array}$ & $\begin{array}{l}15,49 \\
(1,41)\end{array}$ & $\begin{array}{r}66,26 \\
(14,51)\end{array}$ & $0,654^{* *}$ \\
\hline $\begin{array}{l}\text { Universidade de Coimbra } \\
(n=1597)\end{array}$ & $\begin{array}{l}14,47 \\
(1,14)\end{array}$ & $\begin{array}{r}66,10 \\
(14,27)\end{array}$ & $0,655^{* *}$ \\
\hline $\begin{array}{l}\text { Faculdade de Medicina da Universidade do Porto } \\
(n=1472)\end{array}$ & $\begin{array}{l}14,74 \\
(1,07)\end{array}$ & $\begin{array}{r}69,95 \\
(14,33)\end{array}$ & $0,679^{* *}$ \\
\hline $\begin{array}{l}\text { Instituto de Ciências Biomédicas Abel Salazar da Universidade } \\
\text { do Porto } \\
(n=972)\end{array}$ & $\begin{array}{l}13,34 \\
(1,03)\end{array}$ & $\begin{array}{r}67,30 \\
(13,89)\end{array}$ & $0,586^{* *}$ \\
\hline $\begin{array}{l}\text { Universidade da Beira Interior } \\
(n=309)\end{array}$ & $\begin{array}{l}15,61 \\
(1,01)\end{array}$ & $\begin{array}{r}63,84 \\
(12,47)\end{array}$ & $0,591^{* *}$ \\
\hline $\begin{array}{l}\text { Universidade do Minho (Escola de Ciências da Saúde) } \\
(n=264)\end{array}$ & $\begin{array}{l}15,02 \\
(1,03)\end{array}$ & $\begin{array}{r}72,33 \\
(12,50)\end{array}$ & $0,651^{* *}$ \\
\hline $\begin{array}{l}\text { Universidades e Escolas Medicas Estrangeiras } \\
(n=1107)\end{array}$ & $\begin{array}{l}12,56 \\
(2,56)\end{array}$ & $\begin{array}{r}48,85 \\
(15,16)\end{array}$ & $0,236^{* *}$ \\
\hline Total $-n=8956$ & & & 0,576 ** \\
\hline
\end{tabular}


língua Portuguesa (PALOP e Brasil) 27,3\% $(n=207)$.

Por outro lado, cerca de $6,5 \%$ dos Portugueses tinham-se licenciado no estrangeiro, formando a larga maioria $(43,1 \%)$ do grupo com Licenciatura Internacional. O seu número aumentou em valor absoluto todos os anos de 42 em 2007 para 174 em 2011.

A nota de Licenciatura era, em média, 14,73 $\pm 1,66$ (numa escala de 0 a 20 valores), não seguia uma distribuição normal e aumentou ao longo dos anos, de 14,4 em 2006 para 14,9 em 2011. A média dos médicos licenciados em Portugal era 15,04 $\pm 1,21$ e a dos Licenciados no estrangeiro de 12,56. Esta última apresentava um pico nos 10 valores, o que poderá resultar do sistema de equivalência aplicado (Fig. 1).

As médias de Licenciatura não eram iguais entre as Faculdades Nacionais e distribuíam-se em 4 grupos significativamente diferentes (ANOVA, $F=645,88(7,8948), p$ $<0,001$ ) (Tabela 2): a) a FMUL, UBI e FCM apresentavam as médias de curso mais altas $(15,5$ - 15,7), não diferindo entre si de forma significativa. Seguiam-se b) a UM e c) a FMUP e depois d) com as médias mais baixas, a FMUC e o ICBAS, que também não diferiam entre si. A diferença máxima entre as escolas era de 1,37 pontos.

Os candidatos internacionais em média, tinham menos três valores do que os Licenciados em Portugal e maior variação.

\section{Análise univariada da Classificação obtida no ENS}

A nota obtida no teste foi, em média, de $65,54 \%$ ( \pm $15,72)$ e não seguia uma distribuição normal quer com todos os candidatos quer apenas os Licenciados em Portugal. A nota média sofreu variações significativas ao longo dos anos (Anova $F=31,35(5,8950), p=0,000$ ), entre $68,5 \%$ em 2007 e $62,45 \%$ em 2011, o que pode traduzir diferentes graus de dificuldade do teste. Cerca de $2 \%$ dos candidatos obtiveram classificações muito baixas, iguais ou inferiores a $30 \%$, distribuindo-se um pouco por todas as faculdades (Figura 2).

Embora existissem diferenças estatisticamente significativas $(F=266,73(7,8948), p<0,001)$ de média entre as Escolas Médicas Nacionais, a análise pós hoc mostrou que várias Faculdades não diferiam entre si, a diferença média entre escolas era de poucos pontos, sendo o máximo 8,49 pontos o que corresponde a oito ou nove perguntas do teste (Tabela 2). A comparação entre as Faculdades Nacionais é apresentada na Tabela 3, sendo de notar que as escolas que se encontram na mesma coluna não diferem significativamente entre si.

Os médicos de Nacionalidade Portuguesa obtiveram, em média, uma classificação 22 pontos superior à dos candidatos internacionais $(67,41$ vs 45,21 ) (Student $t=-40,23$ $p=0,000,95 \% \mathrm{Cl}:-23,154 ;-21,002)$ e as médicas mais um ponto do que os médicos $(65,99$ vs 64,77$)$ (Student $t=$

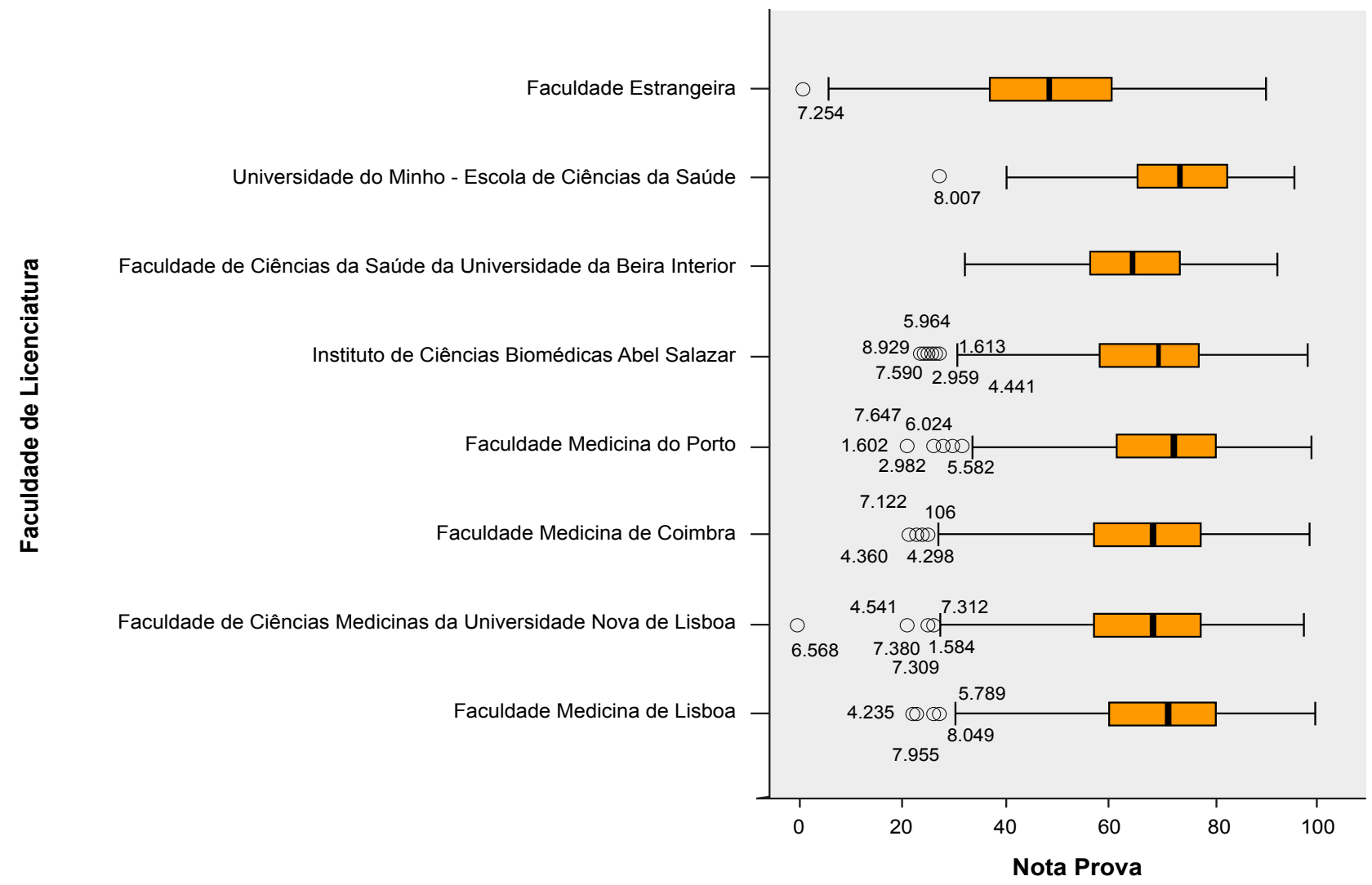

Figura 2 - Distribuição das notas do exame por Faculdade de Licenciatura/Mestrado Integrado, com indicação dos outliers. 
$-3,45, p=0,000,95 \% \mathrm{Cl}: .523 ; 1902)$. A nota dos licenciados no estrangeiro foi inferior à dos diplomados em Portugal $(48,85 \pm 15,16$ vs $67,98 \pm 14,31)$ (Student $t=-33,40, p$ $<0,001,95 \% \mathrm{Cl}-19,99 ;-18,0)$. Por outro lado, os médicos de nacionalidade Portuguesa licenciados no estrangeiro, obtiveram a classificação média de $54,3 \%$ [com uma variação anual entre 52 (2011) e 59\% (2007)], ou seja superior à média dos outros licenciados no estrangeiro.

Verificou-se ainda que existia uma correlação positiva e significativa (Pearson $r=0,576, p<0,000$ ) entre a nota do ENS e a de licenciatura. Esta correlação era mais forte na FMUP $(r=0,679)$ e na FMUC $(r=0,655)$ e mais fraca nos Licenciados Internacionais $(r=0,236 . p<0,000)$ (Tabela 2).

\section{Analise multivariada}

O modelo de regressão linear múltipla da nota do exame em função das variáveis analisadas (ano do exame, género, nacionalidade Portuguesa ou estrangeira, faculdade e média de Licenciatura), entre os licenciados em Portugal, foi estatisticamente significativo $(F(5,7843)=1003,62$; $\left.\mathrm{R}^{2}=0,39 ; p<0,001\right)$ e explicava $39 \%$ da variabilidade da nota do ENS. A análise dos coeficientes de regressão mostrou que todas as variáveis consideradas se associavam à nota no ENS, particularmente a nota de Licenciatura, seguida da Faculdade de Licenciatura e depois o ano do exame.

Por outro lado, quando se consideraram todos os candidatos, o modelo explicava $38 \%$ da variabilidade na classificação do ENS $\left(F(5,8950)=1111,28 ; R^{2}=0,383 ; p<\right.$ 0,001 ) e a nota de Licenciatura, o ano e a nacionalidade Portuguesa eram as principais variáveis preditivas mas não tanto a Faculdade de origem (Tabela 4).

\section{DISCUSSÃO}

Neste estudo procurámos caracterizar o perfil académico dos médicos que se candidataram ao Exame Nacional de Seriação num período de seis anos e compreender os factores que determinaram a sua classificação nesta prova. Destacamos os seguintes achados:

1. Existem marcadas assimetrias no número de licenciados que se formam nas diferentes Faculdades Nacionais e por conseguinte no número de candidatos de cada escola ao exame de seriação, com maior peso das três Universidades mais antigas do país, a FMUL a FMUC e a FMUP. Estas diferenças de proporção foram consistentes ao longo dos seis anos analisados.

Por outro lado, a percentagem de médicos com licenciaturas internacionais que se candidatam ao ENS tem vindo a aumentar, constituindo $15 \%$ dos candidatos nos dois últimos anos analisados. Em 2011 o seu número ultrapassou não só o dos candidatos da UM e da UBI mas também do ICBAS, da FCM e da FMUP.

Verificou-se ainda que um número crescente dos Licenciados no estrangeiro é constituído por Portugueses, que provavelmente tentam regressar ao país durante a formação pós graduada. Embora não tivéssemos informações que permitissem organizar estes candidatos por Faculdade de Licenciatura, os seus dados são muito relevantes tanto mais que este grupo tem vindo a aumentar. A sua análise pode ajudar a conhecer a formação que os estudantes de Medicina Portugueses têm recebido no estrangeiro, comparar o seu desempenho e o seu trajecto profissional com o dos médicos saídos das Faculdades Nacionais e até contribuir para compreender a adequação do actual método de selecção para a formação pré graduada praticado em

Tabela 3 - Análise pos hoc de Tukey HSD das notas médias no ENS por Faculdade de origem. Os grupos que se encontram na mesma coluna não diferem significativamente entre si.

Subset for alpha $=0,05$

\begin{tabular}{|c|c|c|c|c|c|c|c|}
\hline \multirow{2}{*}{ Faculdade de Licenciatura } & \multicolumn{7}{|c|}{ Subset for alpha $=0,05$} \\
\hline & $\mathbf{N}$ & 1 & 2 & 3 & 4 & 5 & 6 \\
\hline Faculdades Estrangeiras & 1107 & 48,85 & & & & & \\
\hline Faculdade de Ciências da Saúde da Universidade da Beira Interior & 309 & & 63,84 & & & & \\
\hline Faculdade de Medicina de Coimbra & 1597 & & 66,10 & 66,10 & & & \\
\hline Faculdade de Ciências Médicas da Universidade Nova de Lisboa & 1400 & & & 66,26 & & & \\
\hline Instituto de Ciências Biomédicas Abel Salazar & 972 & & & 67,30 & 67,30 & & \\
\hline Faculdade de Medicina de Lisboa & 1835 & & & & 69,41 & 69,41 & \\
\hline Faculdade de Medicina do Porto & 1472 & & & & & 69,95 & \\
\hline Universidade do Minho - Escola de Ciências da Saúde & 264 & & & & & 72,33 & \\
\hline Sig. & & 1,000 & 0,064 & 0,778 & 0,107 & 0,997 & 1,000 \\
\hline
\end{tabular}


Portugal.

2. Os candidatos Internacionais ao ENS compreendem mais de 50 nacionalidades diferentes, o que ilustra bem a progressiva diversidade de cultura, língua e formação dos médicos que vêm prosseguir o seu treino em Portugal. Existe seguramente uma enorme heterogeneidade de motivos que os levam a candidatar a este exame, com factores de atracção por Portugal e outros de afastamento dos países de origem, tal como tem sido identificado noutros trabalhos. ${ }^{6}$ É provável que neste grupo existam grandes diferenças de idade, tempo desde a licenciatura, empenho na formação avançada e nos motivos da emigração, que podem explicar a variabilidade de resultados que este grupo obtém na prova.

Embora a maioria destes candidatos provenham de países de Língua Portuguesa, há também muitos médicos do Leste da Europa e da União Europeia, de países integrados no processo de Bolonha, que poderão ter uma formação Universitária mais homogénea relativamente à Portuguesa. Segundo um estudo sobre a mobilidade dos profissionais de saúde na Europa, ${ }^{7}$ o número de profissionais estrangeiros a exercer em Portugal triplicou entre 1998 e 2004, tendo havido posteriormente um decréscimo relacionado com a saída de muitos médicos Espanhóis. De acordo com a mesma fonte, em 2007 os principais grupos vinham de Espanha, dos PALOP ou do Brasil, e metade dos médicos estrangeiros já tinham uma especialidade. Nesta análise esses grupos foram suplantados pelos médicos da Ucrânia e é possível que para alguns médicos, este exame seja a primeira tentativa de obtenção de um posto no Serviço Nacional de saúde, mas não dispomos desses dados.

3. Existem diferenças pequenas, mas significativas, nas médias de curso entre as Escolas Nacionais, que podem indicar diferentes critérios de avaliação ou discrepâncias nas características dos alunos. Por outro lado existe uma tendência para o aumento das médias ao longo do tempo.
A média de curso foi a variável mais preditiva da nota no ENS e o grau de correlação entre as duas dependia da universidade.

Se considerarmos a nota de Licenciatura como o gold standard da avaliação, uma vez que representa uma média de todas as avaliações ao longo de seis anos e em todos os domínios e competências (conhecimentos, atitudes, gestos, comunicação), esta correlação mostra que apesar de todas as limitações do ENS, a sua nota não é completamente imprevisível. Os alunos com melhor preparação tendem a obter classificações mais altas e os com pior desempenho ficam mais mal classificados. Esta correlação não valida o conteúdo nem o constructo do ENS mas poderá ser considerada uma medida de validade de critério, ou validade convergente, que habitualmente é definida pela correlação entre variáveis. ${ }^{8,9}$

É importante sublinhar que este teste avalia apenas conhecimentos e dentro de uma área restrita da medicina interna. Uma formação mais centrada no raciocínio clínico, atitudes, gestos e na comunicação não tem tradução nesta nota e pode ser penalizada nesta avaliação..$^{10} \mathrm{O}$ facto das variáveis incluídas no modelo de regressão só explicarem $39 \%$ da variação do ENS significa que há outros factores a condicionar a nota, um dos quais pode ser a qualidade, o tempo e o esforço de preparação para o exame. A ausência de dados dos candidatos anteriores à Licenciatura (média de entrada para a Faculdade, por exemplo), ou referentes à classificação em disciplinas próximas das avaliadas neste teste, constitui uma limitação desta análise e não nos permite distinguir o efeito do candidato do da aquisição de conhecimentos durante o curso.

Será interessante comparar as correlações encontradas nesta análise com as do novo teste de seriação, que supostamente irá abranger mais conteúdos e contemplar a capacidade de raciocínio clínico e análise de casos, sendo previsível que se venha a correlacionar de forma mais

Tabela 4 - Análise de regressão linear múltipla

\begin{tabular}{|c|c|c|c|c|c|c|}
\hline Nota no ENS total dos candidatos & $\begin{array}{c}R^{2} \\
0,383\end{array}$ & $\begin{array}{c}\text { Adjusted } R^{2} \\
0,383\end{array}$ & Beta & $T$ & $p$ & $95 \% \mathrm{Cl}$ \\
\hline Género & & & 0,017 & 2,054 & 0,04 & 0,$025 ; 1,094$ \\
\hline Nacional. Portuguesa & & & 0,180 & 18,55 & 0,000 & $9.085 ; 11,232$ \\
\hline Faculdade de Licenciatura & & & 0,066 & 6,614 & 0,000 & 0,$327 ; 0,602$ \\
\hline Ano do exame & & & $-0,175$ & $-20,74$ & 0,000 & $-1,699 ;-1,406$ \\
\hline Nota de Licenciatura & & & 0,544 & 53,362 & 0,000 & 4,$963 ; 5,342$ \\
\hline $\begin{array}{l}\text { Nota no ENS considerando apenas os Licenciados } \\
\text { em Portugal }\end{array}$ & 0,625 & 0,390 & & & & \\
\hline Género & & & 0,054 & 6,02 & 0,000 & 1,$076 ; 2,115$ \\
\hline Nacional. Portuguesa & & & 0,037 & 4,05 & 0,000 & 1,$828 ; 5,251$ \\
\hline Nota de Licenciatura & & & 0,641 & 66,1 & 0,000 & 7,$378 ; 7,830$ \\
\hline Ano do exame & & & $-0,147$ & $-16,52$ & 0,000 & $-1,331 ;-1,049$ \\
\hline Faculdade de Licenciatura & & & 0,188 & 20,29 & 0,000 & 1,$482 ; 1,800$ \\
\hline
\end{tabular}


estreita com a nota de Licenciatura, a aumentar o valor preditivo do modelo ou mesmo a alterar as correlações encontradas dentro de cada escola, dependendo do sistema de ensino e das eventuais estratégias de aprendizagem que possam estimular. ${ }^{11-13}$

4. Outro aspecto de relevo foi a comparação de desempenho entre os candidatos provenientes das diferentes Escolas Médicas Portuguesas. Embora existam diferenças estatisticamente significativas entre algumas delas, a diferença máxima entre Universidades correspondia a oito ou nove perguntas nas 100 do exame, e a alguns décimos entre outras. Esta pequena amplitude de variação, associada às diferentes percentagens de alunos estrangeiros em cada escola, sugere que a formação dos médicos nas diferentes universidades do país, pelo menos no que respeita aos conhecimentos em Medicina Interna, não é muito discrepante.

Esta proximidade de resultados contrasta com a distância de pontuações obtidas entre os Licenciados Nacionais e os diplomados no estrangeiro, que apresentaram uma enorme variação de resultados e pouca correlação com a média de curso, o que torna a sua nota muito mais imprevisível. De facto, entre os licenciados internacionais a média de licenciatura explica apenas $5 \%$ da variância no teste.

A compreensão dos resultados obtidos pelos candidatos estrangeiros carece pois de uma análise mais fina que permita identificar, por um lado, o local e a qualidade da formação que receberam e, por outro, os motivos da vinda para Portugal e as dificuldades específicas que possam sentir na adaptação à língua, cultura, à avaliação e até á organização do sistema de saúde e princípios éticos e jurídicos da prática clinica no nosso país. Esses aspectos têm impacto no seu contacto com as populações.

Entre todos os licenciados internacionais que falavam Português, os médicos Portugueses foram os que obtiveram a melhor classificação no ENS, ainda assim abaixo das escolas nacionais, seguindo-se os médicos Moçambicanos e depois os do Brasil.

É muito interessante notar que os médicos estrangeiros que se licenciaram em Portugal seguem mais de perto o padrão dos médicos portugueses, reduzindo o peso da nacionalidade (Portuguesa ou estrangeira) na nota da prova, o que pode significar uma maior familiaridade com o método de avaliação e com o grau de desenvolvimento científico e tecnológico da Medicina que é exigida neste teste, entre outros factores.

5. Finalmente é de assinalar que existem candidatos, praticamente de todas as escolas médicas, que obtêm notas muito baixas no teste, inferiores a $30 \%$, o que poderá corresponder a respostas ao nível do acaso. Até à data, as pontuações baixas não têm tido qualquer tipo de consequências, talvez por não se saber qual o valor a dar a esta classificação, nem se conhecerem as propriedades métricas do teste (consistência interna, validade de constructo, grau de dificuldade, etc.). Na verdade, se considerarmos que o teste existe há mais de 30 anos e é realizado todos os anos, sobre a mesma matéria e no mesmo formato de perguntas de escolha múltipla, isso pode significar que já se esgotou o pool de perguntas sensatas e de relevância clínica e que não se sabe qual a percentagem de perguntas sensíveis à formação clínica.

Neste estudo não tivemos acesso a dados que ilustrassem o trajecto profissional destes candidatos, nem sabemos se prosseguiram treino pós graduado, nem que especialidadeescolheram ou se não escolheram.

De qualquer modo, a existência de desempenhos muito baixos poderá justificar que, no futuro, se estabeleça um benchmark ou uma medida de desempenho mínimo para a prática clínica, de forma a garantir a qualidade e a segurança durante o treino pós graduado, tal como se tem instituído em muitas áreas da Medicina. ${ }^{14}$

Em países onde a formação dos médicos é muito heterogénea e diversificada, como por exemplo no Reino Unido, onde $37 \%$ dos médicos no activo são estrangeiros, ${ }^{15}$ tem sido usado o método de Angoff ${ }^{16-18}$ na sua acreditação. De acordo com esta metodologia, um grupo de peritos identifica as perguntas do teste a que um médico de nível médio deve saber responder para poder exercer com segurança e estabelece-se depois um valor 'de corte' para essas perguntas, que pode ser da ordem dos 80 ou $90 \%$ de respostas correctas. Desta forma, um exame pode produzir duas classificações distintas. Uma para seriar candidatos (com a pontuação total) e outra para excluir candidatos (com a pontuação nas perguntas nucleares). Esta seria uma forma de garantir que só seguem para a formação pós graduada candidatos com o mínimo de conhecimentos nucleares de base, tendo que se encontrar soluções ou formas de recuperação para os Licenciados mais fracos e compreender os motivos deste insuficiente desempenho.

\section{CONCLUSÃO}

O exame de acesso à especialidade é uma oportunidade única para estimular a aquisição de conhecimentos por parte dos médicos recém-licenciados a nível nacional. Uma selecção criteriosa de conteúdos poderá fazer desse exame uma mais-valia para o Serviço Nacional de Saúde, além da sua utilidade na seriação dos candidatos. É também uma oportunidade para comparar desempenhos a nível nacional.

Existem padrões no número e no desempenho dos alunos provenientes das diferentes Faculdades Nacionais e um aumento crescente de candidatos com Licenciaturas Internacionais. As variáveis identificadas neste estudo explicam entre 38 e $39 \%$ da variância da nota do exame, verificando-se uma correlação estreita entre a média de curso e a nota no ENS.

Dada a tendência actual de incorporar diferentes competências na avaliação, e de utilizar a avaliação na promoção da aprendizagem, parece-nos importante que o futuro exame oriente os candidatos para áreas relevantes da prática clínica, para as necessidades de saúde da população Portuguesa e que inclua a avaliação de outras competências, além dos conhecimentos. Poderá constituir uma forma de garantir a qualidade e a segurança durante a forma- 
ção pós graduada, altura em que os médicos começam a assumir progressiva autonomia e responsabilidade.

Prevendo-se que o futuro ENS seja mais bem estruturado e abrangente que o actual, pensamos que será importante que também seja sistematicamente analisado de modo a poder monitorizá-lo, validá-lo e adaptá-lo aos novos desenvolvimentos da Ciência e da tecnologia, o que aliás constitui uma recomendação internacional. ${ }^{19}$

\section{AGRADECIMENTOS}

Agradecemos a Filipe Magalhães (Departamento de Planeamento e Gestão de Recursos Humanos na Saúde - DPGRHS DRH), pelo envio dos dados e a João Carvalho das Neves da ACSS pela autorização para a sua divulgação; a Pedro Custódio e Inês Mares pela ajuda na conversão de ficheiros de dados; a Benjamin Ohana, Algelika Lubanova, Patricia Lobo, e Tiago Mestre que nos fornece-

\section{REFERÊNCIAS}

1. Longo D, Fauci A, Kasper D, Hauser S, Jameson J, Loscalzo J, editors. Harrison's Principles of Internal Medicine. $18^{\text {th }}$ Ed. New York: McGraw Hill; 2011.

2. Cilliers FJ, Schuwirth LW, Adendorff HJ, Herman N, van der Vleuten $\mathrm{CP}$. The mechanism of impact of summative assessment on medical students' learning. Adv Heal Sci Educ. 2010;15:695-715.

3. Cilliers FJ, Schuwirth LW, van der Vleuten CP. A model of the pre-assessment learning effects of assessment is operational in an undergraduate clinical context. BMC Med Educ. 2012;12:9.

4. Chapman DM, Calhoun JG. Validation of learning style measures: implications for medical education practice. Med Educ. 2006;40:576-83.

5. IBM SPSS Statistics Inc. Statistical Package for Social Sciences 21.0. Chicago:IBM;2012.

6. Tjadens F, Weilandt C, Ecker J, the MoHProf consortium. Mobility of Heath professionals. Health Systems, work conditions and patterns of health workers' mobility and implications for policy makers'. Brussel: European Commission; 2012. [Consultado em 2013 Jan 17] Disponível em: http://www.mohprof.eu/LIVE/DATA/National_reports/national_report_Summary.pdf.

7. Conceição C, Ribeiro JS, Pereira J, Dussault G. Mobility of Health Professionals [Consultado em 2013 Jan 17] Bonn:MOHprof;2011. Disponível em: http://www.mohprof.eu/LIVE/national_reports.php.

8. Urbina S. Essentials of Psychological Testing. New Jersey: John Wiley \& Sons; 2004

9. Andreatta PB, Gruppen LD. Conceptualising and classifying validity evidence for simulation. Med Educ. 2009;43:1028-35.

10. Egener B, Cole-Kelly K. Satisfying the patient but failing the test. Acad ram informações detalhadas sobre os sistemas de acesso à especialidade noutros países, e a Marta Gonçalves pela ajuda na bibliografia. Agradecemos ainda a João Ferreira pela análise crítica do texto.

\section{CONFLITOS DE INTERESSE}

Os autores declaram a inexistência de conflitos de interesse na realização do presente trabalho.

\section{FONTES DE FINANCIAMENTO}

Não existiram fontes externas de financiamento para a rea lização deste artigo.

Comunicação apresentada no Colóquio "O Futuro Exame de Entrada na Especialidade e o Ano Comum", Organizado pelo Conselho Pedagógico da Faculdade de Medicina, Universidade de Lisboa, a 18 de Dezembro 2012.

Med. 2004;79:508-10.

11. Schmidt HG,Vermeulen L, van der Molen HT. Long term effects of problem-based learning: a comparison of competencies acquired by graduates of a problem-based and a conventional medical school. Med Educ. 2006;40:562-7.

12. Tiwari $A$, Lai $P$, So $M$, Tiwari $A$, Lai $P$, So $M$, et al. $A$ comparison of the effects of problem-based learning and lecturing on the development of students' critical thinking. Med Educ. 2006;40:547-54.

13. Curry L. Cognitive and learning styles in medical education. Acad Med. 1999;74:409-13.

14. Ben-David MF. AMEE Guide No. 18: Standard Setting in Student Assessment. Med Teach. 2000; 22:120-30.

15. Slowther A, Hundt GL, Taylor R, Slowther A, Lewando Hundt GA, Purkis $\mathrm{J}$, et al. Experiences of non-UK-qualified doctors working within the UK regulatory framework: a qualitative study. J R Soc Med. 2012;105:15765.

16. Angoff $\mathrm{WH}$. Technical problems of obtaining equivalent scores on tests. J Educ Meas. 1964;1.

17. Tiratira N. CutOff Scores: The basic Angoff Method and the Item Response Theory Method. Int J Educ Psychol Assess. 2009;1:39-47.

18. Downing SM, Tekian A, Yudkowsky R. Procedures for establishing defensible absolute passing scores on performance examinations in health professions education. Teach Learn Med. 2006;18:50-7.

19. Ben-David MF. Principles of assessment. In: Dent JA, Harden RM, editors. Pratical Guide for Medical Teachers. $2^{\text {nd }}$ ed. Oxford: Elsevier Churchill Livingstone; 2005.p.282-92. 